\section{Antidepressant use (during pregnancy) and miscarriage}

We thank Einarson ${ }^{1}$ for her commentary on our study on the risk of spontaneous abortion associated with antidepressant use. ${ }^{2}$ Although it is true that we cannot assess causality based on one observational study owing to the inherent potential biases, repetition of findings, which is a strong tenet of epidemiology, leads to cause. At least one other study, by Einarson and colleagues, ${ }^{3}$ has shown an almost identical increase in risk. We disagree that case-control studies, as opposed to cohort studies, have a confusing array of statistics. Both designs, in population-based cohorts, use similar statistics. The cohort design suggested by Einarson is not efficient, and perinatal pharmacoepidemiologists would agree that the case-control approach is the best design for studying adverse perinatal events. We also disagree with Einarson's comment that our adjustment for underlying indication was impossible. We included a group of women with (a) depression who were not using antidepressants, (b) depression who were using antidepressants and (c) no depression. With rates of depression during gestation increasing, it is imperative that we fully understand the full spectrum of risks and benefits of antidepressant use for mothers and fetuses.

\section{Anick Bérard PhD}

\section{Hamid Reza Nakhai-Pour MD PhD}

University of Montreal, CHU Ste-Justine, Montréal, Que.

Perrine Broy MSc

Rennes, France

\section{REFERENCES}

1. Einarson A. Antidepressants and pregnancy: complexities of producting evidence-based information. CMAJ 2010:182;1017-8.

2. Nakhai-Pour HR, Broy P, Bérard A. Use of antidepressants during pregnancy and the risk of spontaneous abortion. CMAJ 2010;182:1031-7.

3. Einarson A, Choi J, Einarson TR, et al. Rates of spontaneous and therapeutic abortions following use of antidepressants in pregnancy: results from a large prospective database. J Obstet Gynaecol Col 2009;31:452-6.

For the full letter, go to: www.cmaj.ca/cgi/eletters /100507v1

DOI:10.1503/cmaj.110-2084

\section{Raising money for cystic fibrosis: At what price?}

Every morning in the metro, we are exposed to the "Drowning on the Inside" advertisement from the Canadian Cystic Fibrosis Foundation (CCFF). The ad is in so many public places, it is hard to avoid it (www.cysticfibrosis.ca/assets/files/pdf /Print_Ad_MagE.pdf). The ad depicts a child, literally drowning. It reads, "Cystic fibrosis is like drowning on the inside. You cough. You gasp. Your lungs fill with fluid. Every breath is a struggle. That's how you live. And how you die." With this ad, money is raised for cystic fibrosis $(\mathrm{CF})$ research, but at the expense of tremendous harm to people with the disorder and to their families.

Living with CF is not easy. Does 14-year-old Karen need to stand in front of this advert with her first boyfriend? It is difficult enough at her age for Karen to have to expectorate her sputum every day and to realize that she'll one day have to do it in the presence of her partner. It is hard enough for her to explain to her friends that, although she has a serious illness, she is okay right now. Later, when her boyfriend leaves her, does Karen wonder if it is because he thought she would die soon? And what about Dorothy, who has to bring her sensitive eight-year-old son Sam to the psychologist after he sees this ad? He is convinced that his mother lied to him because "she wants him to be happy." What about Lisa and Alan, both CF carriers who elected against prenatal diagnosis? Do they also need to be exposed to this kind of misinformation?

Pediatricians are tasked with announcing the diagnosis of $\mathrm{CF}$ to families, many of whom have no knowledge about the disease other than what they've seen in the metro or on the side of a bus - pictures of a young child drowning. One of the goals of the CCFF is to promote public awareness. Living with the disease is not like drowning; dying with the disease may sometimes be like drowning. Children with CF do not gasp. Dying patients gasp. Another of the ads from CCFA shows a straw: "That's what breathing is like with cystic fibrosis. No wonder so many people with CF stop breathing in their early 30s" (www.cysticfibrosis.ca/assets/files /pdf/BreathingCCFF_VertEng.pdf).

I once saw two adolescents standing in front on this ad; they took their straws from their McDonalds cups and had a contest to see who would last the longest in the "CF challenge."

"Wow," said one. "I guess they get really good at it if they do it all the time, poor guys. I would rather die than have to breathe like this."

CF is not like breathing through a straw, at least not the straw shown in this ad.

With advances in medical care, patients born today live much longer than they did 30 years ago. It is ironic that we can find this information on the CCFA site: "[about patients born in 2006] the medical age of survival for Canadians with CF has increased 10 years since 2002 and is currently estimated to be at 47.7 years of age, which is quite remarkable" (www.cysticfibrosis .ca/assets/files/pdf/CPDR_ReportE.pdf).

The patients' own foundation uses this language, not out of ignorance, but with full knowledge of the facts - just to make money. The goals achieved with the money raised are not worth the misinformation and the harm done to vulnerable patients and their families.

\section{Annie Janvier}

Department of Pediatrics, University of Montreal, Montréal, Que.

DOI: 10.1503/cmaj.110-2082

\section{Lifetime care for patients with autism}

The model of care in Nova Scotia proposed by Casey ${ }^{1}$ is spot on, but the scope of the project is faulty.

The unrelenting pressure for resources for autism services and research tends to bury at least two important facts. First, many children 
are challenged by complex neurodevelopmental disorders, and all would benefit from a properly coordinated and accessible system of intervention and support services. It would be unconscionable if discrepancies existed among children with different kinds of cancer - full support for those with bone cancer but meagre support for those with kidney cancer.

Second, it is true that autism and related disorders are diagnosed in more and more children, but the spectrum is broad. The needs of some children and families are complex, but others have fewer needs. Allocation of resources based on a medical diagnosis ignores this crucial fact.

Many professionals involved in the support of children with neurodevelopmental disorders are advocating for child and family support based on need rather than diagnosis. What we really need are regional total care centres for children with complex neurodevelopmental disorders - all those with complex learning and behavioural problems that elude a simple diagnosis.

\section{Anton R. Miller}

University of British Columbia, Division of Developmental Pediatrics, BC

Children's Hospital, Vancouver, BC

\section{REFERENCE}

1. Casey Q. Nova Scotia contemplates a continuum of coordinated, lifetime care for autism patients. CMAJ 2010;182:E391-2.

For the full letter, go to: www.cmaj.ca/cgi/eletters /109-3274v1

DOI:10.1503/cmaj.110-2083

\section{Pharmacogenetic screening}

Fernando and Broadfoot raise interesting points with respect to the potential value of pharmacogenetic screening for prevention of severe adverse drug reactions as well as the possibility of a potential common pathophysiology of drug hypersensitivity syndrome. ${ }^{1}$ There are conflicting theories as to the pathogenesis of drug hypersensitivity syndrome. The reactive metabolite hypothesis assumes elevated concentrations of toxic reactive drug metabolites serve as the syndrome trigger and has led to clinical applications, including testing potentially hypersensitive patients with the lymphocyte toxicity assay and the in vitro platelet toxicity assay.

We have used both assays to test a patient of Han Chinese origin who is HLA-B*1502 positive and developed Stevens-Johnson syndrome (SJS) after starting a course of carbamazepine (CBZ). The test was negative three and nine months after patient recovery, despite a typical CBZ-SJS reaction. At the same time, the test was positive in a patient who developed a nonbullous type of drug hypersensitivity syndrome to carbamazepine. This finding strongly suggests that carbamazepine-induced severe bullous reactions have different underlying pathophysiology than carbamazepine-induced nonbullous reactions. We agree that genetic testing of potential drug hypersensitivity syndrome patients could be a useful approach to minimize morbidity and mortality of such reactions. However, we believe that until a clear understanding of this disorder's pathophysiology is available, a robust cause-effect relationship between such genetic markers and the disease is not achievable.

\section{Abdelbaset A. Elzagallaai MSc and colleagues \\ Departments of Physiology and \\ Pharmacology and Pathology, Schulich \\ Medicine and Dentistry, the University of \\ Western Ontario, London, Ont.}

\section{REFERENCE}

1. Fernando SL, Broadfoot AJ. Prevention of severe cutaneous adverse drug reactions: the emerging value of pharmacogenetic screening. CMAJ 2009; 182:476-80.

For the full letter, go to: www.cmaj.ca/cgi/eletters /182/5/476\#318339

DOI:10.1503/cmaj.110-2062

\section{Paramedic-driven research}

In every medical specialty, and most aspects of health care, research drives improvements in care and systems. ${ }^{1}$ Paramedicine is an emerging health profession. Paramedics have become increasingly involved in conducting important emergency medical services (EMS) research. This has led to publication of scholarly articles changing clinical and operational practice. In Canada, paramedic researchers have struggled to find the right home base to pursue EMS research. It is time to establish a national framework to propel paramedic-driven research forward. Formal training, mentorship, protected time and funding opportunities are essential to the success of paramedic researchers. EMS services must work collaboratively with governments, base hospitals, universities, and other stakeholders to fund paramedic clinicianresearchers; this infusion of support will result in a burst of scholarly work specific to paramedic practice that will inform and improve clinical practice and operational performance.

\section{Blair L. Bigham MSc ACPf}

St. Michael's Hospital, RESCU York

Region Emergency Medical Services,

Toronto, Ont.

Jan L. Jensen MSc ACP

Dalhousie University, Division of EMS

Emergency Health Services, Halifax, NS

Ian E. Blanchard MSc, EMT-P

Alberta Health Services, Emergency Medical Services, Calgary, Alta.

\section{REFERENCE}

1. Casey Q. National trauma divide must be narrowed. CMAJ 2010;182: E295-96.

For the full letter, go to: www.cmaj.ca/cgi/eletters /182/7/E295\#362116

DOI:10.1503/cmaj.110-2079

\section{Research training and residents}

A key point highlighted by $\mathrm{H}$. Savolainen comments ${ }^{1}$ on M. Laberge's article $^{2}$ is the lack of physicians trained in research and conducting interdisciplinary/translational or clinical research. There is an urgent need to train young clinician scientists. Sharing experiences and information on measures used to attract young physicians to research programs is of special interest. This is the practice in France, particularly in pediatrics. Funding is central. France has a national program granting a one-year research training fellowship for resident physicians. Societies, including the French Pediatric Society, organize annual competitions to fund young physicians to maxi- 